\title{
The COMPASS Event Store
}

Venicio Duic, Vladimir Frolov, Ulrich Fuchs, Benigno Gobbo, Massimo Lamanna, Anna Martin, and Marcin Nowak

\begin{abstract}
COMPASS, the fixed-target experiment at CERN studying the structure of the nucleon and spectroscopy, has collected over 500 TB during 2002 and 2003 runs. At the beginning of the experiment these data together with the reconstructed events information were put in a database infrastructure based on Objectivity/DB and on the hierarchical storage manager CASTOR. Starting from 2003 Oracle has been adopted as the database technology. The experience in the usage of the databases is reviewed, and the evolution of the system outlined.
\end{abstract}

Index Terms-Computing in high-energy physics, database management systems, data management, spectroscopy, elementary particles, high-energy data stores.

\section{INTRODUCTION}

C OMMON muon proton apparatus for structure and spectroscopy (COMPASS) [1], [2] is a fixed-target experiment which embraces an extensive physics programme at the CERN SPS using different configurations, in particular using both muon and hadron beams in the $100-300 \mathrm{GeV}$ range at very high intensities. The experiment started taking data in summer 2001, and during the summers of 2002 (the first full physics run) and 2003 it has collected deep-inelastic scattering data for the measurements of $\Delta \mathrm{G} / \mathrm{G}$ of the Deuteron and to investigate transversity effects.

In this paper we will focus on the experience of the first two years of physics data acquisition, comparing the design figures with the actual achievements and elaborate on a roadmap for the future. The value of COMPASS experience in computing is not limited to the experiment itself since it is also a testing ground for key LHC technologies.

\section{COMPASS OFF-LINE SYSTEM}

The off-line system was built to meet severe design constraints, namely a continuous high data acquisition rate (about $40 \mathrm{MB} / \mathrm{s})$ and a very large data sample $\left(10^{9}\right.$ events, $30 \mathrm{kB}$ each, $300 \mathrm{~TB} / \mathrm{year}$ ) to be reconstructed virtually on-line.

The large volume of data to be processed (during a few months each year) and the need for a flexible software environment to cope with the different configurations and measurements of the experiment pushed the COMPASS Collaboration to build a dedicated facility for the off-line computing, the COMPASS computing farm (CCF) [3], and

Manuscript received November 14, 2003; revised May 14, 2004.

V. Duic and B. Gobbo are with the Istituto Nazionale di Fisica Nucleare (INFN), 234127 Trieste, Italy (e-mail: duic@ @ts.infn.it).

V. Frolov is with the Università di Torino, dipartimento di Fisica Generale, 1 10125 Torino, Italy.

U. Fuchs, M. Lamanna and M. Nowak are with CERN, the European Organization for Nuclear Research, 1211 Geneva, Switzerland.

A. Martin is with the Istituto Nazionale di Fisica Nucleare (INFN), 234127

Trieste, Italy. She is also with the University of Trieste, 234127 Trieste, Italy.

Digital Object Identifier 10.1109/TNS.2004.832645 to design the off-line analysis software from scratch, which brought forth CORAL [4], the COMPASS reconstruction and analysis framework, written in $\mathrm{C}++$, providing an analysis class library, interfaces for the event reconstruction algorithms and insulation layers to access the data and for external pluggable packages.

COMPASS decided to build a central data recording (CDR) System $^{1}$ to record all the data: the on-line system does not write the events on tape at the experiment site, but sends them over a few kilometers of dedicated optical fiber network to the computer center, where the CCF, the tape servers, and the corresponding high-speed tape drives are located.

In its original design, during CDR, all the data were entered in a database infrastructure based on Objectivity/DB: the original event files were the input of a procedure that populated the database hierarchically, to ease the navigation during reconstruction and analysis.

The main feature of that database implementation was that to each event corresponded a small object called a header that kept all the basic information on that event, including the RAW data database pointer to the RAW event and multiple versions of the reconstructed objects (referred to as data summary tapes (DSTs), after the name of the dedicated files in which they are stored).

Though recently a new database solution has been adopted-based on Oracle - the basic storage access philosophy has not been changed. Now the CDR takes care to transfer the acquired data directly (in its original format) to CASTOR [5], the CERN hierarchical storage manager (HSM), and contextually produces the corresponding meta-data files for Oracle, which are in fact the headers (held within the database) through which the RAW events can still be accessed (as further described in Section IV-D). The events are now kept in binary files outside the Oracle database (in the following this fact is referred to as the hybrid solution).

These headers (depicted in Fig. 1) still allow to connect at run time all the components of an event (for example, the association between the DST and the corresponding RAW event is kept on a event-by-event basis). The headers are always on-line, while the files containing the RAW and DST events are controlled by CASTOR. Access to full events is therefore made transparent: the possible cases of a program accessing local data, data on a file on a remote server, or recalling data from tape are not exposed in the user software.

At the time of the proposal the estimated computing power to reconstruct all the events at the same speed of the data acquisition was 20000 SI2K (SpecInt 2000 is a standardized CPU performance benchmark [6] designed to measure and compare

${ }^{1}$ It is based on the original CERN CDR, but implemented and managed by COMPASS. 


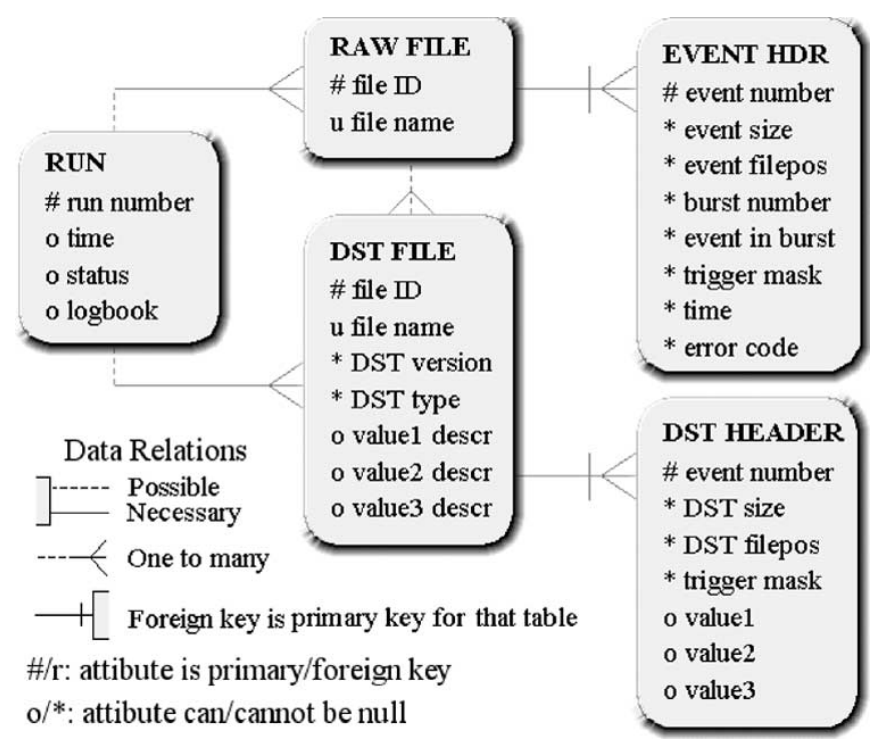

Fig. 1. Relational schema for the new storage system under Oracle.

compute-intensive integer performance. E.g: $1 \mathrm{GHz}$ PIII platform scores 440 SI2K.). The actual figure has grown to above 100000 SI2K in the last versions of the reconstruction program, due to the complexity and the evolution of the event reconstruction. This CPU capacity is provided by some 300 Linux Dual-CPU PCs out of the CERN shared batch system (the total capacity is of the order of $650000 \mathrm{SI} 2 \mathrm{~K}$ ). The choice for the network technology is Gigabit and Fast Ethernet. A disk pool of a few TB has been set up, initially made up of SCSI disks; in production, less expensive EIDE disks have been used.

\section{COMPASS DATA TAKING}

In 2002, COMPASS took data for 12 weeks: after an initial phase of detector and data acquisition commissioning, the rate from the experiment was considerably higher than the design value. Peaks in the rate over $4 \mathrm{~TB} /$ day of files being written to tape have been observed, resulting in a total of $270 \mathrm{~TB}$ of RAW data.

In the 15 weeks of 2003 data acquisition the system has shown to be able to cope with rates well above the higher peaks of 2002 (up to $6 \mathrm{~TB} /$ day-see Fig. 2) but the total recorded size (about $260 \mathrm{~TB}$ ) did not exceed the one of 2002 due to a lower duty cycle of the accelerator system and experiment; in addition, the data of 2003 have a better compression factor.

The CDR system can be described as follows: the on-line system groups the events in files (1 GB maximum, about 30000 events a file) named run chunks. These files flow in parallel multiple streams to the off-line system via the RFIO protocol [5] to the CCF. The transferred files are temporarily held on disk to undergo information extraction in order to populate the meta-data tables of the Oracle database. The files are contextually registered in the CASTOR name space: from this moment onward, CASTOR controls thoroughly the events data handling (copy to tape, managing of the disk space, recall of databases present only on tape).

In the Objectivity/DB solution, the system maintained the original files in a number of disk buffer areas on both the on-line and the off-line farms. In the Oracle implementation, the meta-data extraction is performed directly on the on-line farm so that one transfer step is avoided. The files are deleted after a successful copy to tape has been produced.

Recently, an on-line event filtering has been adopted. This system, still under tests, runs before the events are written into the data-files. In the present implementation stage, the events are rejected if they carry insufficient kinematical information. This currently skims away $20 \%$ of the events.

\section{DATABASE EXPERIENCES}

Since CERN has decided to terminate the support contract for Objectivity/DB with Objectivity Inc, COMPASS was forced to adopt a new solution for its data storage: Oracle $9 \mathrm{i}$ has been chosen to be the new DBMS.

\section{A. Migration of Data From Objectivity/DB to Oracle}

The planning for the migration of COMPASS data from the Objectivity/DB-based storage system to a new, Oracle-based solution had started in the summer 2002.

The first stage was to design the new database system following the lines outlined further in Section IV-D. The next stage was an implementation of the actual migration program that would read existing Objectivity files and produce the corresponding flat files with the events, while storing the event headers in the Oracle database. It is worth noting that the reading part of the migration program could be drawn from the existing CORAL code, while the writing part became the first implementation of the new, Oracle-based CORAL version.

The initial tests of the migration program performed on a single, standard CERN PC disk server with dedicated tape drives showed an average performance of $10 \mathrm{MB} / \mathrm{s}$. To be able to migrate all COMPASS RAW data of 2001 and 2002 (about 300 TB) in less than three months, the migration had to run at about $100 \mathrm{MB} / \mathrm{s}$, which implied using several parallel streams. The three month period was the time window between the moment the new output tape drives (9940B) were made available for COMPASS, and the start of the 2003 data taking.

To achieve the desired migration speed, a dedicated migration cluster was set up. The cluster consisted of 25 PC disk servers, 10 input tape drives and eight output tape drives (the new model of the output tape drives-9940B - was faster than the older 9940). The disk servers were under the control of CASTOR - 11 nodes were reading and processing Objectivity/DB data, 14 nodes were writing the produced output files. Event headers were stored in three separate Oracle databases. Additionally, special software had to be developed for controlling the cluster and managing the migration process. The actual migration started in December 2002. Initially, only a small fraction of the cluster was made available, due to resource shortages. The missing elements were added gradually. The software controlling the migration was also improving as more experience was gained. Both factors were increasing the migration speed and in the last month the daily throughput was over 8 TB.

The migration was performed by the CERN IT/DB group [7]. Fig. 3 shows the migration performance over time. 


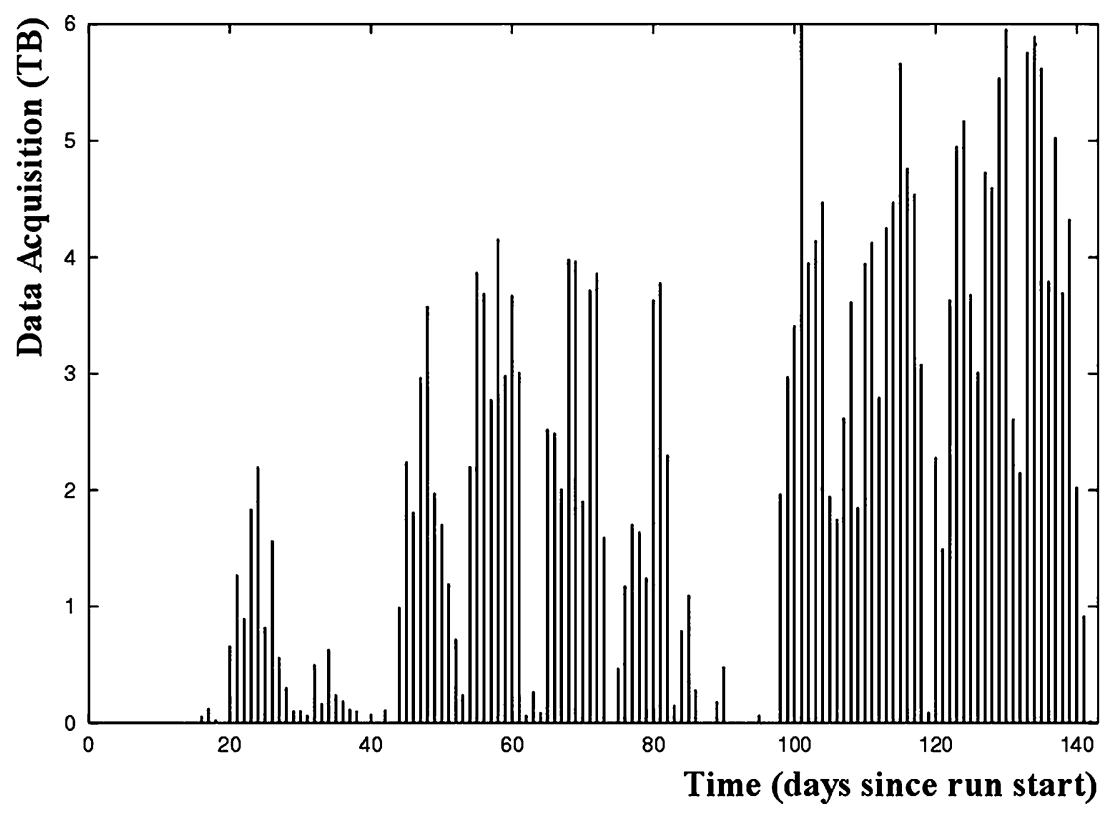

Fig. 2. Data acquisition rate for 2003 DAQ activity. The size of the data transferred per day by the CDR system from the on-line farm to tape is shown.

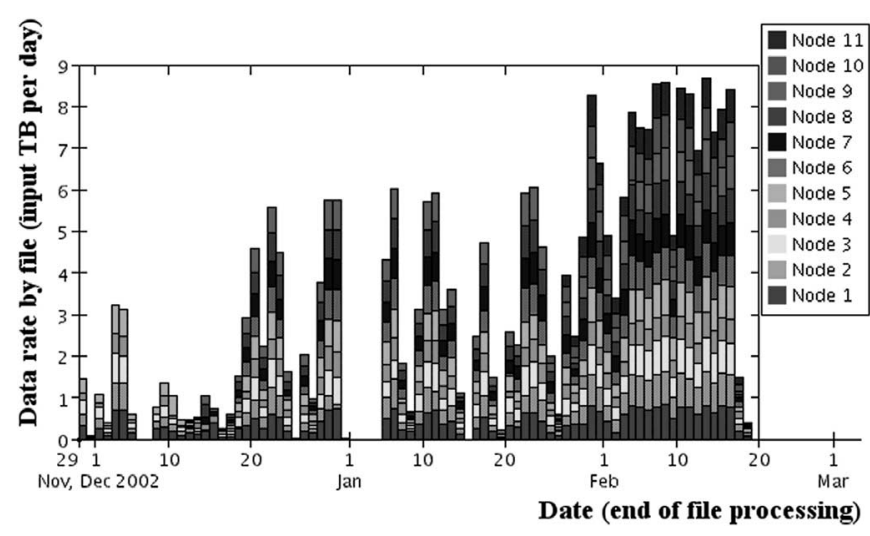

Fig. 3. COMPASS data migration performance (data rate per real time).

\section{B. Oracle Tests}

The migration of the COMPASS data has been validated by a series of checks aimed at verifying that the new system was correctly set up and working, and tests carried on in order to have a preliminary picture of the new system, as soon as a minimal number of RAW data were migrated.

Consistency checks have been performed by COMPASS on basically $100 \%$ of the data for the first samples (comparing each event from the Oracle $9 \mathrm{i}$ and the Objectivity/DB stores). Globally, about $5 \%$ of the data have been selected randomly across the full sample to verify data integrity.

The functionality of the new store has been verified by joint activities of COMPASS and the CERN IT/DB group.

Stress tests have been undertaken accessing the RAW data for reading by a variable number of concurrent clients, while the concerned storage system components (Oracle 9i, and RFIO daemons), CPU and network loads were monitored, to get information on how the system responded to the strain. This kind of access reproduces the typical access pattern in COMPASS, though a simultaneous start of many clients was expressly introduced to stress the system as much as possible.
As far as the users point of view is concerned, the performance obtained in scanning the data contents, measured by the time spent to scan a given amount of data, as a function of the concurrent accessing clients, has shown a nice scalability of the implemented system, up to about 100 concurrent clients.

Besides the scalability properties, the new system has also proven to be able to provide the clients with the requested data at a speed that was as close as the network bandwidth could afford (we recall that the data are read via the very efficient RFIO protocol, since they are kept in a flat file format out of the database). The new system did not show any limitation arising from the server itself, in contrast to what we had observed for Objectivity/DB.

The information further drawn from monitoring the system, demonstrated that, thanks to the adoption of the hybrid system, the data access load is shared between the DBMS and the RFIO daemons because, as soon as the Oracle server locates the requested data-file (and the relevant data-block entry point), the data intensive transfer is performed by a RFIO daemon on the data-server hosting that file.

The very good performances observed in scanning large sets of data are inherited from the RFIO system which is optimized for this use case; to every active client corresponds an RFIO daemon serving the data over the network.

\section{Objectivity/DB Experience}

The strong points COMPASS observed in operating large $\mathrm{Ob}-$ jectivity/DB federations were the following:

- The separation between the logical and the physical layer assured independence from the HSM details;

- The central role and the straightforward manageability of the catalogue as a repository for databases physical location handling, allowed us to integrate the product with the HSM in a clean way; in particular, it was possible to balance in a transparent way the load on the different machines serving as the CASTOR disk cache; 
- The client-driven nature of Objectivity/DB has shown to be suited to the access pattern of COMPASS data handling, which resulted in the possibility of achieving a high degree of concurrence in production (up to 400 concurrent clients); the AMS (Advanced Multithreaded Server: the network database server in Objectivity/DB parlance) is designed to deal with many concurrent clients via a set of lightweight stateless processes.

- A simple but effective file protection (against accidental errors) could be put in place in the CERN version of the AMS (purely based on user UID). This was sorely needed since Objectivity/DB tools do not implement any user access policies management.

On the other hand, the experience with that database management system (DBMS) has lead COMPASS to deal with the following drawbacks:

- Many transactions were not automatically rolled back in case of common client failures: the recovery from this situation was a major burden for the database administrators. To ease the clean-up, the generic users accessed the federation in read-only mode. This feature is implemented via the reconstruction program, whilst a centralized access control system would have been very useful.

- The clean-up of aborted transactions turned out to be really complex; in some cases, a restart of the Lock Server is the only viable solution, which on the other hand leads all user applications to abort. The removal of locks becomes a problem when more than 100 clients are accessing the database.

- Objectivity/DB Lock Server and AMS can be effectively operated as "1 box per process and per database federation", but the available COMPASS resources compelled the collaboration to make some compromise to this requirement.

- Database creation is a particularly delicate operation that exhibits an imperfect "atomic" nature, and might lead to update locks that lock the whole federation. These locks are extremely dangerous because they prevent any data from being written (COMPASS could continue to take data though, thanks to the CDR multiple buffers: once the lock is removed, the CDR can catch up by sending data to tape at higher speed). The resolution of these problems required expert intervention, which it was not possible to automate because it relied on unpublished options of Objectivity/DB tools. The regime where these problems are likely to appear is well above $40 \mathrm{MB} / \mathrm{s}$, and a few database created per minute (in this situation the central infrastructure holding the database catalogue was $100 \%$ busy dealing with these operations).

- Objectivity/DB introduces an overhead in the database files of about $30 \%$ of the RAW data size. But once on tape, due to compression, it turns into a factual $6 \%$ overhead (of the corresponding compressed size of the RAW data). This effect was known, but it has to be kept in mind because of financial consideration on the recording media costs.

\section{Oracle Experience}

The technology choice and the implementation of the Oraclebased database system (devised at CERN by the IT/DB group and validated by COMPASS) take into account the two main lessons learnt in building and operating the previous system. First of all, Oracle $9 \mathrm{i}$ as an infrastructure shows the very good feature of error resiliency, making the interaction with it relatively simple from the user point of view; the CERN IT/DB experience in running Oracle-based services plays an important role also. The second lesson is that once the meta-data are in the database, the bulk of the data can be addressed simply via filename and file offset (this was also the back-up solution for the Objectivity/DB store before its scalability properties had been proven).

Unlike for Objectivity/DB, COMPASS is not primarily contributing to the management of the database infrastructure, which is provided exclusively by CERN IT/DB division.

The whole RAW data store under Objectivity/DB has been migrated (as described in the next section) to the new store based on Oracle. The meta-data corresponding to the RAW data of 2002 and 2003 amount to about 1 TB.

No direct DST database migration has been performed but the data of 2002 have been fully reconstructed under the new database infrastructure, since COMPASS has profited from the DBMS migration to improve the format of the DSTs, and to improve on calibrations and algorithms. The produced DSTs are "addressed" by some 150 GB meta-data in the corresponding Oracle DST tablespace.

The reconstruction has the granularity of a physical run-chunk (a typical run is made of about 100 files), and is carried on in parallel, by some 600 jobs, running in a dedicated batch queue. The load of the involved Oracle server during reconstruction is minimized by opening an Oracle session and reading all the relevant events headers into memory (this process takes about $10 \mathrm{~s}$ ). When the reading is done, the production program closes the session and starts the reconstruction, giving the other programs the chance to connect to the database. As soon as the reconstruction of the chunk is completed, a session is opened again and the DST meta-data are written into the database (this process also takes about 10 s). In this way, during the few hours chunk reconstruction, only a few concurrent Oracle sessions happen to occur, thus minimizing database access contention.

One of the main advantages of the hybrid solution is that the data flow can be substantially simplified: basically, the RAW data are just transferred to the CASTOR system directly from the on-line system. This also cuts by a factor of two the traffic inside the CASTOR disk servers front-end, and the effective disk space. During the transfer to CASTOR, the meta-data are extracted and written on auxiliary files (typically $1.5 \mathrm{MB}$ in size). These files are asynchronously read, and their content is used to create the Oracle 9i meta-data entries. Since the system is made of two almost independent components, the RAW data can be recorded also during a database outage or a database maintenance period, which was simply impossible in the Objectivity/DB store solution.

The other advantage of the hybrid oracle-based system is the better performance, which, as emerged from the stress tests outlined in Section IV-B, descends principally from the data access load sharing between the DBMS and the RFIO daemons. The 
performance is furthermore enhanced in case of large datasets scanning, due to the specific RFIO optimization.

The only limitations observed so far are the following:

- Queries selecting a large number of events tend to overload the server (in DST reading, for example a common query selects a few millions of Oracle records); splitting such selections into multiple queries to optimize the performances has solved this problem.

- The better performance of a RFIO daemon (compared to the AMS thread/subprocess of Objectivity/DB) can be cancelled if a single data-server is trying to serve too many clients (due to the relative heavier structure of RFIO in terms of required system resources).

This issue is avoided by a properly designed data-server load balancing in running conditions. In fact during the production of 2003 a set of some 10 data servers could sustain over 600 clients reading at an aggregate speed of more than $20 \mathrm{MB} / \mathrm{s}$, and writing at about $2 \mathrm{MB} / \mathrm{s}$ of data to tape.

As already described, the load on the database servers has been further reduced by effectively shortening the transaction duration.

- The load balance across servers has to be proactively introduced via a load balancing system residing in a library linked to the user code. In the Objectivity/DB store, this parameter was instead explicitly expressed as a property of the databases, therefore independent from any user application.

\section{DATA PRODUCTION}

As the technical run took place in 2001 (collecting few tens of TB), COMPASS managed to submit to production those data in Spring 2002. The data of 2002 were first processed during the run (small samples) whilst a larger production was carried on in the subsequent months. The data production was stopped when the migration of the entire data store to Oracle 9i was started, then it started again under Oracle on the data that had not been produced yet. Finally, the data that had been produced under Objectivity/DB have been re-reconstructed and put under Oracle, so that currently all the DSTs can be fully accessed for the analysis.

As far as 2001 data are concerned, The amount of produced DSTs is relatively modest: only 103 GB have been reconstructed, but these data allowed a study of the apparatus and a search for basic physics signal to be performed. The production involving 2002 data is characterized by much bigger figures:

- 5.7 TB of DSTs have been produced (with some reprocessing) out of $80 \mathrm{~TB}$ of the RAWs under Objectivity/DB.

- 8.1 TB of DSTs have come out from the reconstruction of 170 TB of the RAW data under Oracle.

The DST production for the data of 2002 was performed during February-August 2003. More than $3 \cdot 10^{9}$ events were reconstructed in this period with a mean reconstruction speed of about $0.6 \mathrm{~s}$ per event on a Dual PII $600 \mathrm{MHz}$ PC.

The data analysis in COMPASS is performed by the participating institutes scattered across the world. Some of them have already locally replicated part of the DSTs of their interest in the past and, thanks to the manageability of Objectivity/DB, they have been able to cope with it by themselves. For example, INFN-Trieste has set up a shallow copy of the 2002 federation, provided with some 1.4 TB of the DST data, on which the analysis has been performed using the satellite computing farm-ACID [8]. They are preparing to install a copy of the COMPASS CERN Oracle store as well.

All participating institutes perform their analysis directly on mini DSTs, which are selectively filtered out from the DSTs during the production (they turn out to have a size of about $1 \mathrm{~GB}$ per run-approximately $1 \%$ of the original RAW data). They are stored as ROOT [9] files (no database infrastructure is used to access them), and the analysis is performed by means of PHAST [10], the COMPASS framework for the data analysis at mini DST level, which:

- Provides access to reconstructed events;

- Is the environment for final analyzes;

- Is a tool for further filtering out subsamples of events.

\section{CONCLUSION AND OUTLOOK}

The migration of the full COMPASS data store was successful (access library, new CDR system, legacy data migration). As shown in Fig. 4, COMPASS has completed the second big production phase. The first phase (late 2002) corresponds to the handling of 2002 data via Objectivity/DB. As calibrations and alignment have been improved, the new production is a full reprocessing of the data of 2002, using the Oracle 9i infrastructure. The DST reconstruction of 2003 data is about to start.

The CDR of 2003 data (using Oracle 9i) was successful, and could be run in parallel with DST productions and with occasional further access to the new data.

COMPASS has had an important role as pilot user of the computing infrastructure and services at CERN in many different areas:

- Computing fabric-COMPASS computing power needs are (in production mode) the highest at CERN for analyzing real data, rivaling with the large computing challenges of the LHC experiments [11];

- Data management - the COMPASS data store integrated size is already the largest at CERN [5], besides being the only CERN experiment which used Objectivity/DB in production (real experimental data) to a multihundred TB scale.

- Central Data Recording-COMPASS has the highest rate to mass storage with physics data among the current CERN experiments, close to the expected rate for the multipurpose LHC experiments. For example, the rate in 2003 was equivalent to the one foreseen for CMS in 2007 [11].

To our knowledge, demonstrating a high rate to tape record (peak per day, mean per day, number of days) is unique (for example BaBar [12] populated a larger database, but on a much longer period, namely a few years).

The extensive use of different approaches to data management has yielded very interesting sets of experience as a result of: 


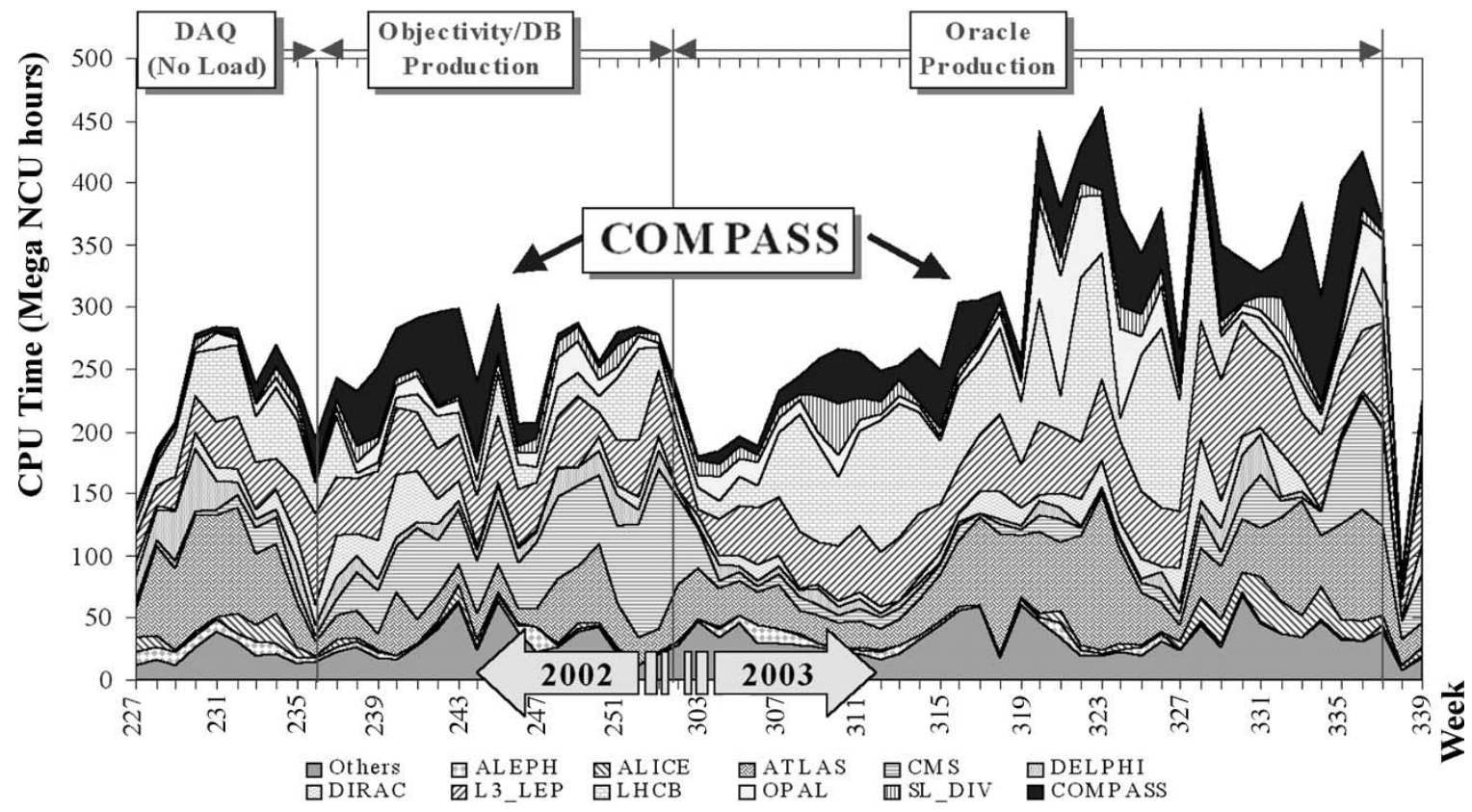

Fig. 4. COMPASS DST production activity (CPU time per week) among CERN experiments (DAQ activity of 2003 is not evidenced as the CPU load is negligible).

- The operation of very large data stores of real data, which well complements the dedicated technical runs (data challenges) using dummy data-the COMPASS experience is at the moment unique in terms of duration (100 days of data taking per year) and total aggregate size;

- The successful change of a database technology within an experiment and the corresponding data migration, with no disruption in the continuity of data access, which has demonstrated the feasibility of such a large data conversion (see for example CHEP2003).

All this experience has contributed in building a solid system for COMPASS, and, as a by-product, a fertile exchange of technological and operational experience at CERN, in the context of which the COMPASS experiment has pioneered data management solutions that CERN is planning and preparing for the LHC experiments. A fruitful intercourse that will continue in the next future.

\section{ACKNOWLEDGMENT}

The authors would like to acknowledge the encouragement and support of the COMPASS collaboration and of the CERN IT division. The authors would like to explicitly thank the CASTOR team, whose enthusiastic support has made it possible to effectively operate such a complex system and the IT/DB group for support all along the evolution of the COMPASS data store system.

\section{REFERENCES}

[1] G. Baum, J. Kyynäräinen, A. Tripet, R. Gehring, S. Goertz, and W. Meyer et al., "COMPASS: A Proposal for a Common Muon and Proton Apparatus for Structure and Spectroscopy," CERN, Geneva, Switzerland, Tech. Rep. CERN-SPSLC-96-14, Mar. 1996.

[2] - "COMPASS Addendum 1 Common Muon and Proton Apparatus for Structure and Spectroscopy," CERN, Geneva, Tech. Rep. CERNSPSLC-96-30, May 1996.

[3] M. Lamanna, "The COMPASS computing farm project," in Proc. CHEP 2000, 2000, p. 576.

[4] The COMPASS Reconstruction and AnaLysis Program (CORAL), COMPASS Collaboration. [Online]. Available: http://coral.cern.ch

[5] The CASTOR Project Page. [Online]http://castor.web.cern.ch/castor/

[6] SPEC Homepage, Standard Performance Evaluation Corporation.http://www.spec.org/index.html [Online]

[7] M. Nowak, D. Düllmann, K. Nienartowicz, A. Valassi, M. Lubeck, and D. Geppert. Objectivity data migration. presented at CHEP03. [Online]http://arxiv.org/ftp/hep-ex/papers/0305/0305097.pdf

[8] ACID: The COMPASS Farm of INFN Trieste, COMPASS Trieste Collaboration.http://www.ts.infn.it/experiments/compass/farmts/ [Online]

[9] ROOT: An Object-Oriented Data Analysis Framework, R. Brun and F. Rademakers. [Online]. Available: http://root.cern.ch/

[10] PHAST: The Physics Analysis Software Tool, COMPASS Collaboration. [Online]. Available: http://ges.home.cern.ch/ges/phast/

[11] M. Delfino and L. Robertson, Eds., (2001, Jul.) CERN-IT-DLO2001-003 Version 1.2-Solving the LHC Computing Challenge. [Online]. Available: http://lcg.web.cern.ch/lcg/PEB/Documents/CERNIT-DLO-2001-03.doc

[12] A. Adesanya, T. Azemoon, J. Becla, A. Hanushevsky, A. Hasan, and W. Kroeger et al.. On the verge of one petabyte-the story behind the BaBar database system. presented at CHEP'03. [Online]. Available: http://arxiv.org/ftp/cs/papers/0306/0306020.pdf 